Revue d'histoire de l'Amérique française

DEV RUE D.HISTOIRE DE L'AMÉRIQUE FRANÇAISE

\title{
Correspondance autour de la question scolaire du Nouveau-Brunswick - 1873
}

\section{Lionel Groulx}

Volume 4, numéro 2, septembre 1950

URI : https://id.erudit.org/iderudit/801638ar

DOI : https://doi.org/10.7202/801638ar

Aller au sommaire du numéro

Éditeur(s)

Institut d'histoire de l'Amérique française

ISSN

0035-2357 (imprimé)

1492-1383 (numérique)

Découvrir la revue

Citer ce document

Groulx, L. (1950). Correspondance autour de la question scolaire du

Nouveau-Brunswick - 1873. Revue d'histoire de l'Amérique française, 4(2),

268-275. https://doi.org/10.7202/801638ar d'utilisation que vous pouvez consulter en ligne.

https://apropos.erudit.org/fr/usagers/politique-dutilisation/ 


\section{DOCUMENTS INEDITS}

\section{CORRESPONDANCE AUTOUR DE LA QUESTION SCOLAIRE DU NOUVEAU-BRUNSWICK - 1873}

Nous commençons la publication de quelques lettres de M. Cléophas Beausoleil à M. Alphonse Desjardins. Elles sont de l'année 1873. Nous en avons déjà fait état dans notre ouvrage: l'Enseignement français au Canada, tome II, Les Ecoles des Minorités. En 1873 la Cour suprême du Nouveau-Brunswick rejetait les revendications des catholiques de cette province. Des écoles catholiques avaient existé au Nouveau-Brunswick, avant 1867, affirmait le tribunal, mais sans le caractère légal qui pat justifier l'intervention du gouvernement d'Ottawa. Cette décision suivait de près le refus d'intervenir, déjà exprimé par le parlement fédéral lui-même. Mais, en 1873, le défenseur attitré de la minorité, M. Costigan, ramenait le débat devant les Communes canadiennes et, pour obtenir justice à ses coreligionnaires, préconisait un amendement à la charte fédérative. Ces quelques faits permettent de situer la correspondance Beausoleil-Desjardins. Correspondance intéressante qui révèle les dessous de l'histoire parlementaire de l'époque. Voici maintenant quelques notes sur les deux correspondants.

Cléophas Beausoleil est né à Saint-Félix de Valois, Comté de Joliette, en 1845. Il a fait ses études au Collège de Joliette. Journaliste, à ce qu'il semble, à l'Événement, il serait passé au Nouveau-Monde en 1868, pour en devenir rédacteur en chef de 1870 à 1873. Cette année-là même, il fonde, avec L.-O. David, le Bien Public. Appelé au barreau en 1880, il devient en 1887 l'un des associés d'Honoré Mercier, puis député de Berthier aux Communes de 1887 à 1899. Il était libéral en politique, mais del'école de Mercier. En 1899 il succède à Arthur Clément-Dansereau, dans les fonctions de maître de poste de Montréal.

Alphonse Desjardins est né à Terrebonne, le 6 mai 1841. Il 6́tudie au Collège Masson et au Séminaire de Nicolet. Admis au bar- 
reau en 1862 il pratique le droit à Montréal jusqu'en 1868. En 1867 il se fait journaliste et fonde le Nouveau-Monde. Il devient député du comté d'Hochelaga aux Communes, puis ministre de la Milice en 1896, dans le cabinet Mackenzie Bowell, et ministre des travaux publics dans le cabinet de Sir Charles Tupper. Défait aux élections de 1896 il se retire de la vie publique. Il meurt le 4 juin 1912. Excellent chrétien, il a été fait, en 1872, chevalier de l'Ordre de Pie IX.

Lionel Groulx, ptre.

(à Alph. Desjardins, Es)

Montréal.

Personnelle

Ottawa, 6 mars 1873

Mon cher Monsieur,

Je suis arrivé ce matin à sept heures, et je vous envoie ce soir une partie - la moindre - des informations que j'ai recueillies. Les autres sont encore confidentielles ou incomplètes. Je puis ajouter que Bellerose me paraît bien prêt de passer à l'Opposition et qu'il s'en donne à cœur-joie sur le gouvernement en général et sur Langevin en particulier. Il regrette beaucoup qu'il n'y ait point d'opposition à Terrebonne. Votre nom est ici dans toutes les bouches et l'on vous verrait avec plaisir entrer au Parlement par la porte qui est maintenant grande ouverte.

Je n'ai fait qu'entrevoir Masson. Je ne sais rien de ses dispositions. Costigan passe à l'opposition et $M$. Renaud, qui conteste le siège de Cutler, m’a déclaré que s'il réussit, il en fera autant. Les catholiques du NouveauBrunswick ont perdu tout espoir de rien obtenir tant que les fanatiques comme Tilley et ses créatures de Fredericton resteront au pouvoir; voilà pourquoi ils veulent amener la chute du ministère actuel, comptant que celuici tombé, ils auraient bientôt raison de l'autre.

Prevost assiste à tous les caucus de l'Opposition et il a déjà reçu son role. Quant à Cauchon je suis sur la piste d'une longue histoire que je vous compterai en détail un de ces jours.

Les maisons de pension sont remplies et je n'ai pas encore trouvé à me loger. Je ne sais encore où je coucherai ce soir. Les prix sont exhorbitans. J'aimerais à être bien au courant de ce qui se passe à Montréal, surtout de ce qui concerne le journal et moi-même personnellement.

M. Genoud (?) vous fait bien des amitiés. Présentez mes hommages au Sr Henri Desjardins, à M. le chanoine et à Laferrière.

Tout à vous,

C. Beausolfil

Chambre des Communes, Ottawa. 
Ottawa, 10 mars 1873.

Cher Monsieur,

Comme vous me donnez des nouvelles de nos bons québecquois, je fais de même.

Tous nos amis les gallicans sont dans la joie - depuis Langevın jusqu'à Elie Tassé inclusivement. On a fait courir le bruit que l'on avait reçu de Rome des nouvelles annonçànt que l'archevêque avait gagné sur toute la ligne, et Cauchon qui ne fait jamais les choses à demi, annonçait - ce qui est vrai sans doute - que l'archevêque avait enjoint aux journaux de Québec de se taire absolument sur les questions religieuses. Et il ajoutait - ce qui est faux - que le Nouveau-Monde avait aussi reçu un Monitum sévère, lui enjoignant de cesser toute discussion religieuse. Ne sachant comment concilier cet ordre avec la conduite du N.M. il dit que Mgr Bourget a fait mander Mr Lamarche et lui a donné ordre de se taire, ce à quoi notre digne Chanoine aurait repondu qu'il n'en ferait rien et que si l'évêque le forçait à l'obéissance, $i l$ se retirerait sur sa ferme! !!

On voit ici des gens qui avalent cela sans craindre les indigestions!...

Le gouvernement d'Ottawa prend des leçons du gouvernement de Québec. Pour éviter la défaite, il concède à ses adversaires tout ce qu'ils demandent. C'est ainsi qu'il n'a pas osé résister à la motion de l'Opposition pour donner le siège de Muskoka à $M$. Cockburn. S’il y avait eu vote, l'Opposition l'aurait emporté d'une dizaine de voix certainement. L'Hon. M. Blake a engagé sa parole d'honneur que le ministère fédéral serait à terre avant trois semaines. L'opposition déploie une activité, une entente et une adresse dont les ministériels paraissent fort dépourvus. Le sentiment général est que l'heure du décès est venu et dans la députation de Québec, on sera fort heureux si Langevin prend enfin une culbute.

Je ne dis rien, mais je crois que si nos amis voulaient, ils pourraient obtenir de l'Opposition toutes les concessions que Langevin leur a refusées. Masson et Bellerose disent qu'ils soutiendront le gouvernement jusqu'à nouvel ordre ou jusqu'à ce qu'il se présente une bonne occasion de le renverser. Ni l'un ni l'autre ne doute de sa chute prochaine, et ils s'en réjouissent tous deux, bien qu'ils ne veuillent pas y contribuer.

J'ai lu à Masson cette partie de votre lettre qui le concerne, et il m'a dit que cela était bien; whatever it may mean.

$\mathrm{Au}$ sujet de mon traitement, je vous écrirai de nouveau cette semaine. Des saluts à M. Lamarche et à M. Laferrière. Des saluts à M. Genoud (?) à qui il vient d'arriver une aventure que je vous raconterai.

Tout à vous.

C. Beausoleil 
Mon cher Monsieur,

Ottawa, 14 avril 1873

J'ai réfléchi mûrement au projet de paix que notre ami Routhier voudrait conclure entre le Nouveau Monde et le gouvernement. Je crois aujourd'hui comme il y a quatre mois que si une alliance était possible à des conditions honorables, elle serait fort désirable pour l'intérêt de la causè de l'Église et pour celui du parti conservateur. Mais le malheur veut que le chef du Bas-Canada soit un homme faux, égoïste et sans foi, qui flatte d'une main pour mieux frapper de l'autre, qui me fait off rir une situation de $\$ 1,500$. et me fait insulter par ses valets. Si c'était un homme de parole comme M. Cartier, il serait facile de s'entendre. Mais Langevin ne renoncera jamais à sa haine, et s'il fait faire des démarches aujourd'hui, croyez-bien qu'il ne s'agit que de baillonner le Nouveau Monde au moment où les better terms et les écoles vont venir sur le tapis. Déjà il a sondé les membres, surtout ceux qui l'année derinière ont voté en faveur du désaveu du bill des Écoles et il leur a représenté qu'il ne fallait point pour une affaire de sentiment mettre en danger le ministère et la confédération. Il est évident que le dernier acte de la comédie se prépare et que dans quinze jours des catholiques de la province de Québec auront prouvé que pour eux il est beaucoup plus i'nportant de sauver le ministère et de concilier les mécontents du NouveauBrunswick que de sauvegarder les intérêts de leurs coreligionnairess et le grand principe de la liberté de l'Église. Voilà où Langevin veut tous nous amener.

Cependant les négociations n'engagent à rien, tant qu'elles ne sont pas conclues et s'il faut encore une preuve - après celle de l'hiver dernier que l'on veut tout simplement nous jouer un tour et se moquer de notre bonne foi, je n'ai pas la moindre objection à ce que l'on tente une nouvelle épre'ıve. Mais il me semble, que tout en continuant d'appuyer le gouvernement d'une façon générale et de lui donner tout le crédit de nos bonnes œuvres, nous ne pouvons ni ne devons céder sur la question de principe de l'affaire des coles. Il y va de l'honneur du journal, de notre honneur personnel et surtout de la liberté de l'Église.

Je puis vous donner un exemple des bonnes dispositions de Langevin. Mais ceci est absolument confidentiel: "c'est qu'il refuse de nommer $M$. Leclerc chapelain du pénitencier de St-Vincent de Paul, parce qu'il est Directeur du Nouveau Monde et qu'il faut pour cette position un ami dévoué du gouvernement".

Mgr a écrit deux lettres pour le recommander et s'il est nommé c'est que Langevin craindra que ces imprudentes paroles soient révélées au public. Enfin pour me résumer, je dirai que je ne crois pas à la sincérité des démarches du gouvernement, qu'il ne s'agit que de faire le silence pendant que l'on jouera une no'svelle comédie aux catholiques du Nouveau-Brunswick et que la guerre recommencera ensuite plus implacable que jamais.

Je pense aussi que le Nouveau-Monde doit garder une attitude bienveillante à l'égard du pou voir malgré toutes les insultes et toutes les provoca- 
tions, tenir en un mot la ligne de conduite qui était indiquée dans le petit entrefilet intitulé: "Les maladroits". Que vous en semble!

Ce qui n'empêche point que nous pourrions obtenir pour nos principes et pour nos amis toutes les concessions et les garanties que nous pourrions exiger, comme prix d'une alliance avec l'opposition. Mais je vous ai déjà dit et je persiste à croire qu'un journal comme le Nouveau Monde ne peut ni ne doit descendre dans l'arène des disputes de partis, se faire l'avocat attitré du gouvernement ou de l'opposition; qu'il doit avoir des principes dont il ne s'éloigne jamais, s'approchant tantôt de la droite et tantôt de la gauche suivant qu'elles s'approchent ou s'éloignent davantage de ces principes. C'est ainsi que l'entend Mgr de Montréal si j'ai bien compris la conversation que vous m'avez dit avoir eue avec lui quelques jours avant la session.

Vous avez lu sans doute l'article que le Courrier d'Outaouais m'a consacré. J'ai hésité longtemps si je devais faire une réfutation appuyée d'extraits de toutes ces calomnies, ou bien ne rien dire du tout ou bien encore le mettre dans l'alternative de prouver ces avancés, de produire ces pièces compromettantes ou de se rétracter. C'est ce dernier parti que j'ai adopté après considération. $\mathrm{J}_{\Theta}$ ne veux point faire intervenir le $N . M$. dans une querelle personnelle, et je ne vous demanderai de reproduire cette lettre qu'en autant que la Minerve se mettra de la partie ou que le Courrier me fermerait ses colonnes. Dans ce dernier cas une simple note expliquerait la position et tout en resterait là.

Rien de neuf ici, sinon que nos amis sont très heureux des nouvelles reçues de Rome et qu'ils ont applaudi des deux mains à votre article sur Chapleau, qui doit bien regretter son joli tour qui ne tourne point à sa gloire.

Vous aurez vu M. Masson à son passage à Montréal. Informez-moi done, si possible, de ce qu'il pense et de ce qu'il se propose de faire. J'ai toujours compris qu'il maintiendrait énergiquement la position qu'il avait prise l'année dernière.

Veuillez présenter mes amitiés à $M$. Lamarche et à M. Laferrière et me croire.

Votre tout dévoué

C. Beausoleil

Ottawa, 21 avril 1873

Mon cher Monsieur,

Vous voudrez être mis au fait de la question des Écoles et de la position qui est faite à nos amis. Ces explications seront un peu longues, mais je vais tâcher de les donner complètes.

Je dois vous dire tout d'abord que la position a changé depuis que M. Masson est allé à Montréal et qu'il a vu Mgr Bourget. On ne savait 
pas alors ce qui s'était passé en Angleterre et que les officiers en loi de la Couronne, sans attendre les plaidoiries des partis, sans prendre la peine de se rendre compte de la loi ancienne ni de la nouvelle, à deux jours d'avis, avaient concouru dans l'opinion de sir John et du cabinet canadien. On ne savait pas qu'après avoir reçu cette opinion, l'argument du gouvernement local du N. Brunswick avait été transmis aux officiers en loi qui auraient répondu que cela ne changeait point leur manière de voir (beau dommage) et que le mémoire de l'évêque de St Jean ne leur avait pas été communiqué. On ignorait également que le gouvernement avait attendu plus de cinq mois avant de faire aucune démarche dans le but de se conformer aux ordres de la chambre et qu'il n'avait absolument fait aucune représentation au gouvernement local du N.B. Les documents soumis le jour même de son retour ont révélé tous ces faits et cet autre non moins significatif que c'est le lendemain du jour où la motion de Mercier fut adoptée que fut passé l'Ordre en Conseil demandant une nouvelle délibération, comme s'il y avait quelque probabilité que les officiers en loi renverseront leur décision.

Enfin pour mettre le comble à la mesure, la majorité du comité des impressions a décidé que les documents ne pourraient être imprimés pour la présente session. Le gouvernement travaille à éluder la question et à la renvoyer indéfiniment. Il craint le résultat. C'est pour cela que les documents qui pouvaient être préparés en deux jours - c'est presque entièrement composé des mémoires imprimés - n'ont été transmis qu'au bout d'un mois et que maintenant on leur refuse l'impression. C'est encore pour cela que sir John ne fait point venir les lois iniques passées durant la dernière session de la législature locale, qui aggravent si fortement la position de nos coreligionnaires. Je ne serais point surpris que Tilley aurait fait dire aux ministres lòcaux de ne point les expédier pendant que le Parlement est en session. Toujours est-il qu'on ne voit rien venir.

Voilà pour l'apparence extérieure des choses. Maintenant il est question de quatre modes d'opérations: 1o. le premier qui est proné par Mercier, est un vote de censure basé sur le manque de diligence du gouvernement à se conformer aux ordres de la Chambre. Malgré l'exposé écrasant que l'on peut faire du manque de sincérité des ministres, je crois que les conservateurs de notre province voteraient en masse contre une motion qui comporterait non-confiance directe comme celle-là.

2o. M. Costigan a donné avis d'une résolution demandant au Parlement impérial d'amender l'Acte d'union afin de lui faire déclarer que les catholiques du N.B. continueront de jouir des droits et privilèges relativement aux écoles séparées dont ils jouissaient à l'époque de l'Union. Cette motion a le tort de faire affirmer que l'Acte de 1871 était constitutionnel quand la question est devant le Conseil Privé, sur l'appel des catholiques du N.B. Pour cette raison les conservateurs bas-canadiens la rejetteront.

3o. Enfin il y a une autre motion plus pratique et qui s'attaque à quelque chose de saisissable. C'est celle de M. Anglin, qui demandera le désaveu des dernières lois oppressives passées par la législature. Celle-ci ne pourrait 
manquer de rallier tous ceux qui, l'année dernière, ont voté la motion de censure de M. Dorion.

40. Enfin il reste la question des Better Terms, c'est un puissant levier, et s'il y avait dans notre représentation un homme résolu et qui n'aurait point peur de son ombre, il pourrait faire beaucoup pour amener le règlement de toutes les difficul tés. Le grand obstacle, c'est le gouvernement. On craint de renverser le ministère et bien que l'on soit disposé à voter pour son propre compte, on n'ose prendre la responsabilité d'une attitude qui rallierait assez de voix pour réussir.

Pour ma part, il me semble sauf meilleur avis que l'on devrait s'en tenir aux deux derniers moyens: - exiger le désaveu des lois de 1873 et refuser les Better Terms, même sans assigner la véritable raison, afin de faciliter plus tard les négociations s'il y a lieu. Toute l'opposition sans exception est prête à prendre en main la cause des catholiques, pourvu que ceux-ci ne la trahissent pas eux-mêmes; c.a.d. que pour prix du pouvoir les députés d'Ontario sont prêts à assurer aux catholiques leur liberté; mais qu'ils ne feront rien s'ils ne peuvent atteindre un résultat politique comme compensation de leur attitude religieuse.

Telle est la situation, en dernière analyse et dans sa plus entière exactitude.

Mgr Sweeny doit arriver ce soir ou demain matin. J'irai m'inscrire chez lui dès qu'il sera ici et j'ai demandé à $M$. Renaud de me procurer une audience du prélat dès qu'il voudra bien recevoir. Vous comprenez que j'aurai besoin pour vous bien informer, dé connaître ses véritables intentions et les solutions qu'il propose à nos propres difficultés. Je v.ous écrirai, tout au long ce qui sortira de cet entretien. Il n'y aura rien de décisif d'ici à quelques jours. J'ai essayé de rédiger quelque chose dans le sens que vous m'avez demandé pour le Courrier d'Outaouais; mais j'y suis si fort intéressé que j'use de termes trop violents et je vous prie de faire cela vous-même si vous le jugez bon et si vous persistez à croire qu'il importe d'avertir le public que Bous ne voulons plus avoir de rapports avec un polisson.

Je puis vous dire que la lettre de Mgr de Montréal a trouvé autant d'admirateurs que de lecteurs. Le Nouveau Monde a été dévoré tout le jour et les membres sont venus me demander mon numéro qui a passé dans bien des mains. Les gens de Québec mêmes sont d'accord à dire que l'Archevêque a agi comme un idiot et qu'il n'a que ce qu'il mérite. Bien des écailles sont tombées des yeux de bien des gens, et tout le monde, Cauchon le premier, [ se ] récrie "qu'il ne croyait pas que Mgr Tachereau fût un homme de cette espèce". Jugez de ce que cela veut dire. Le fait est que la lettre de Mgr Bourget est un chef-d'œuvre et qu'elle restera comme un monument à sa gloire.

\section{Confidentiel}

Bellerose est furieux. Langevin lui a dit que Mr Leclere ne serait pas nommé chapelain du Pénitencier parce qu'il était ennemi du gouvernement \& m'a montré une lettre qu'il se propose d'adresser à Sir John et à Langevin pour 
les avertir qu'il fait de la cause de M. Leclerc la sienne et que s'il n'est pas nommé, il se fera plus d'un vide dans le parti ministériel. Son cri de guerre est pas d'ostracisme pour les amis de l'évêque de Montréal, ou la mort... du ministère.

Après cela je crois bien que Langevin rentrera sa rancune quitte à se venger dans une occasion plus favorable.

Rien de plus nouveau ici, si ce n'est que vous m'obligeriez beaucoup en m'expédiant un peu d'argent. J'ai été obligé d'emprunter dix piastres de Genoud (?) et je crois que ça le gêne!....

La session finira vers le 10 de Mai, suivant que sir John l'a dit à Piché.

Veuillez présenter mes amitiés à M. le chanoine Lamarche et à M. Laferrière et me croire votre ami tout dévoué.

C. Beausoleil

(à suivre) 